\section{P197 RETROSPECTIVE COHORT STUDY ON PAEDIATRIC APPENDICECTOMIES AND THE USE OF DIAGNOSTIC ULTRASOUND AT UNIVERSITY HOSPITAL LIMERICK}

Felicia Paluck*, Robert M O'Connell, Shona Tormey. University Hospital Limerick, Limerick, Ireland

\subsection{6/archdischild-2019-epa.552}

Background Acute abdominal pain in children remains a common presentation to A\&E departments across Ireland. Appendicectomy remains a common procedure done in children. In this study we aimed to analyse the number of paediatric appendicectomies from August 2016-August 2018 at the University Hospital Limerick (UHL), and the use of imaging in the diagnosis of acute appendicitis.

Methods Using the Hospital Inpatient Enquiry (HIPE) administrative database, all paediatric patients ( $\leq 16$ years) who underwent an appendicectomy at UHL from August 2016- August 2018 were identified retrospectively. Data were collected on each patient as follows: imaging and results, surgery (open or laproscopic appendicectomy) and histology of appendix. Negative appendicectomy rates and ultrasound diagnostic yields were calculated.

Results From August 2016- August 2018, 474 paediatric patients had an appendicectomy at UHL. Of these, 251 were male $(52.8 \%) .373$ patients had histological evidence of appendicitis (78.7\%), 55 (11.6\%) had evidence of lymphoid hyperplasia, enterobius and/or fecolith with no inflammation and $46(9.7 \%)$ had a normal appendix. There were 303 (63.9\%) laparoscopic appendicectomies and 171 (36.1\%) open appendicectomies. Only 156 (32.8\%) patients had ultrasounds. $104(66.7 \%)$ patient ultrasounds were reported inconclusive for appendicitis. $64(61.54 \%)$ of the patients with an inconclusive ultrasound yield had appendicitis.

Conclusions Ultrasound remains a poor imaging modality in diagnosing appendicitis, which may contribute to the high negative appendicectomy rates.

\section{P198 MANAGEMENT OF CHILDREN WITH DOWN SYNDROME}

Zaheera Yusuf*, Farhana Sharif. Midlands Regional Hospital Mullingar, Mullingar, Ireland

10.1136/archdischild-2019-epa.553

Down Syndrome is a chromosomal anomaly that occurs in one in every 546 live births in Ireland. ${ }^{1}$ Children with Down Syndrome require surveillance and support as they may have complex needs, including cognitive impairment, audiological issues, obstructive sleep apnoea, ophthalmic disease, cardiac defects, neurologic dysfunction, gastrointestinal disease, hip dislocation, thyroid disease, and haematological disorders. ${ }^{2}$

We reviewed the management of children with Down Syndrome attending the Mullingar, Co Westmeath Early Intervention Clinic in March 2017. Patients' charts and investigations were reviewed to determine if management was consistent with national guidelines. ${ }^{3}$ We also reviewed management in the context of the more extensive American Academy of Pediatrics Guidelines.

There were 10 patients (4 males and 6 females) attending the Early Intervention Team ranging from 3 months to 5 years of age. Eight patients were diagnosed with Down Syndrome postnatally, one was diagnosed antenatally, and one was unspecified. We identified four main areas where we were not meeting the national guidelines. First, $20 \%$ of children waited over 6 months for an echocardiogram, and $80 \%$ of children never had an electrocardiogram performed. Second, dental advice or infective endocarditis advice was not documented at any visit. Third, there was a delay in referral for ophthalmology assessment for two patients at their $18-24$ months visit. Fourth, referrals to audiology services were variable and delayed up to 3 years of age. Lastly, the AAP advise a more comprehensive assessment of children at their routine appointments. ${ }^{4}$ While half of patients had a coeliac screen organised, patients rarely had an FBC, CRP, or ferritin performed. Vaccinations, including RSV prophylaxis and influenza vaccine, were discussed with two patients. Families were not given cspine advice, or asked about neurological dysfunction or obstructive sleep apnoea.

Our local practice would be improved with dentition review, and early referrals to and reviews by cardiology, ophthalmology, and audiology. We would suggest the introduction of a clinical template to guide appointments, and a review of the AAP guidelines, with a view to consider including their recommendations into Irish practice.

\section{REFERENCES}

1. Irish Health. http://www.irishhealth.com/article.html?con=64. 2006.

2. Bull MJ, and the Committee on Genetics. Clinical Report-Health Supervision of Children With Down Syndrome. American Academy of Pediatrics. Pediatrics Volume 128 (2): 393-406, 2011.

3. Down Syndrome Ireland. Suggested Schedule of Health Checks. https://downsyndrome.ie/wp-content/uploads/2017/01/Medical-Management-Guidelines-SuggestedSchedule-of-Health-Checks.pdf. 2017.

4. Voigt RG, Macias MM, and Myers SM. AAP Developmental and Behavioural Paediatrics. American Academy of Pediatrics, 2011.

\section{P199 HUDDLING FOR SAFETY: THE FIRST IRISH PAEDIATRIC SAFE COLLABORATIVE}

${ }^{1}$ Rachel MacDonell ${ }^{*},{ }^{2}$ John Fitzsimons, ${ }^{3}$ Peter Lachman, ${ }^{1}$ Alf Nicholson. ${ }^{1} R C P I$, Dublin, Ireland; ${ }^{2}$ HSE, Dublin, Ireland; ${ }^{3}$ ISQUA, Dublin, Ireland

10.1136/archdischild-2019-epa.554

Situation Awareness for Everyone (SAFE) is a collaborative programme developed by the Royal College of Paediatrics and Child Health UK to support clinical teams to improve communication, build a safety based culture and deliver better outcomes for children and young people.

The programme includes Quality Improvement (QI) theory, situation awareness methodology and planning of safety huddles on the wards. It has been implemented in over 50 teams across the UK and the Quality Improvement Department of the Royal College of Physicians of Ireland are delighted to bring this initiative to Ireland.

A National Paediatric Early Warning System (PEWS) was endorsed by the Irish Minister for Health in December 2015. The supporting National Clinical Guideline recommends that hospitals 'support additional safety practices that enhance the PEWS and lead to greater situation awareness among clinicians and multidisciplinary teams, such as incorporating briefings, safety pause and huddles into practice'. Programmes such as SAFE that use quality improvement methods and patient safety science were specified as appropriate to assist hospitals to collaborate in addressing these challenges.

11 consultant-led teams from across Ireland are participating in this SAFE Collaborative. Teams consist mostly of 